\title{
Trust in E-Health System and Willingness to Share Personal Health Data
}

\author{
Dasa BOSANAC ${ }^{\mathrm{a}, 1}$ and Aleksandra STEVANOVIC ${ }^{\mathrm{bc}}$ \\ ${ }^{a}$ University North, Croatia \\ ${ }^{\mathrm{b}}$ University of Rijeka Faculty of Medicine, Croatia \\ ${ }^{\mathrm{c}}$ University of Rijeka Faculty of Health Studies, Croatia
}

\begin{abstract}
Electronic personal health records (ePHR) are web-based tools that enable patients to access their personal health data. Since the data in PHR are systematized, they can be used in scientific research with the patient's consent. Despite the potential benefits of using ePHR, their adoption in Croatia remains low. Multiple factors are influencing the use of PHR and willingness to share personal health data (PHD). The purpose of this paper was to determine familiarity with the e-health system among Croatian citizens, trustfulness in the system itself, and willingness to share PHD with physicians or researchers. Results showed that $34 \%$ of respondents use ePHR, and have less confidence in the electronic system than doctors' confidentiality. However, health professionals have lover trust in doctors' confidentiality compared to non-health workers. Respondents rated mental health data and STD data as most sensitive but are overall willing to share that data with doctors and researchers.
\end{abstract}

Keywords. E-health, personal health records, data sharing, trust, privacy

\section{Introduction}

E-health contributes to improving the efficiency of health services, enables the communication between the patient and the doctor, and ensures the continuity of medical care. Electronic personal health records (ePHR) enable data systematization for easier scientific research [1,2]. However, to provide appropriate services and conduct research through health applications and e-health services, users' consent is required [3].

Previous studies examined factors that influence the frequency of use of personal health data (PHD) and willingness to share them for scientific research. Generally, people are more willing to share their ePHD if they perceive them useful for public health research, not sensitive, and if they trust the anonymity of research [3,4]. The intention to use an ePHD application is strongly affected by the social impact [5], by the ease of usage of the system [6,7], while age, gender, education, and profession showed different influences on the frequency of e-health use and trust in the system itself $[1,6,7]$.

In Croatia, there is an e-Health Portal since 2016 and a Mobile e-Health Portal since 2017, which in addition to basic services, enables citizens to allow or prohibit

${ }^{1}$ Corresponding Author: Daša Bosanac, University North, Trg dr.Zarka Dolinara 1, 48000 Koprivnica, Croatia; E-mail: dabosanac@unin.hr. 
family doctors, dentists, gynecologists, and pediatricians to access a specific PHD. It also provides insight into who and when viewed which data. Considering previous research, we were interested in how much people in Croatia are familiar with the eHealth system, how much they trust such a system, how sensitive they consider specific medical data, and depending on that, how much of the content of the ePHD are they willing to share with doctors or for research purposes.

\section{Methods}

We conducted the research using an anonymous online survey on the KwikSurveys platform. Data were collected in July 2021. We asked respondents if they use the eHealth system and how much they trust e-data and family doctor confidentiality on a scale from 0 to 10 . Furthermore, we asked participants to assess the perceived sensitivity of 13 different health data selected for the study and their willingness to share them, both on a scale from 0 to 10 . Finally, we collected demographic data (age, gender, occupation, self-assessment of digital literacy).

Data are presented as mean and standard deviation or number and percentage. Differences were assessed by chi-squares or t-test for independent groups. Dependent on the type of data, correlations were assessed by either Pearson or Spearman correlation coefficients.

\section{Results}

Out of a total of 198 questionnaires started, 102 were sufficiently completed for analyses ( $62 \mathrm{~F}, 35 \mathrm{M}, 5$ other), average age $\mathrm{M}=43.04$ (range $25-75$ ), $\mathrm{SD}=10.89$. In the sample, $92 \%$ of the participants were employed, of which $37 \%$ were health professionals. $88 \%$ of the sample was highly educated. On a linear scale from 1-10, respondents rated their digital literacy as $\mathrm{M}=7.79, \mathrm{SD}=1.63$.

A total of $73 \%$ of participants reported they were aware of the e-Health Portal's existence in Croatia, yet only $34 \%$ were using it, and $38.2 \%$ were familiar with its possibilities. Confidence in e-Health data protection averaged $6.23, \mathrm{SD}=2.43$, and confidence in the family doctor's confidentiality averaged 7.49, $\mathrm{SD}=2.65$. Participants have significantly greater confidence in doctor's confidentiality than online data protection $(\mathrm{t}=4.603, \mathrm{p}<0.001)$. Also, $61 \%$ of participants reported that family doctors should have complete insight into all patients' medical data. Participants rated their digital literacy as $\mathrm{M}=7.8$ ( $\mathrm{SD}=1.63$, range $1-10)$. Digital literacy significantly correlated with trust in e-Health data protection $(\mathrm{r}=0.27, \mathrm{p}=0.007)$ but not with doctor's confidentiality $(\mathrm{r}=0.09, \mathrm{p}=0.395)$.

Employment status, educational level, gender, and age were not significantly related to the e-Health Portal awareness, use, or knowledge of its possibilities. 


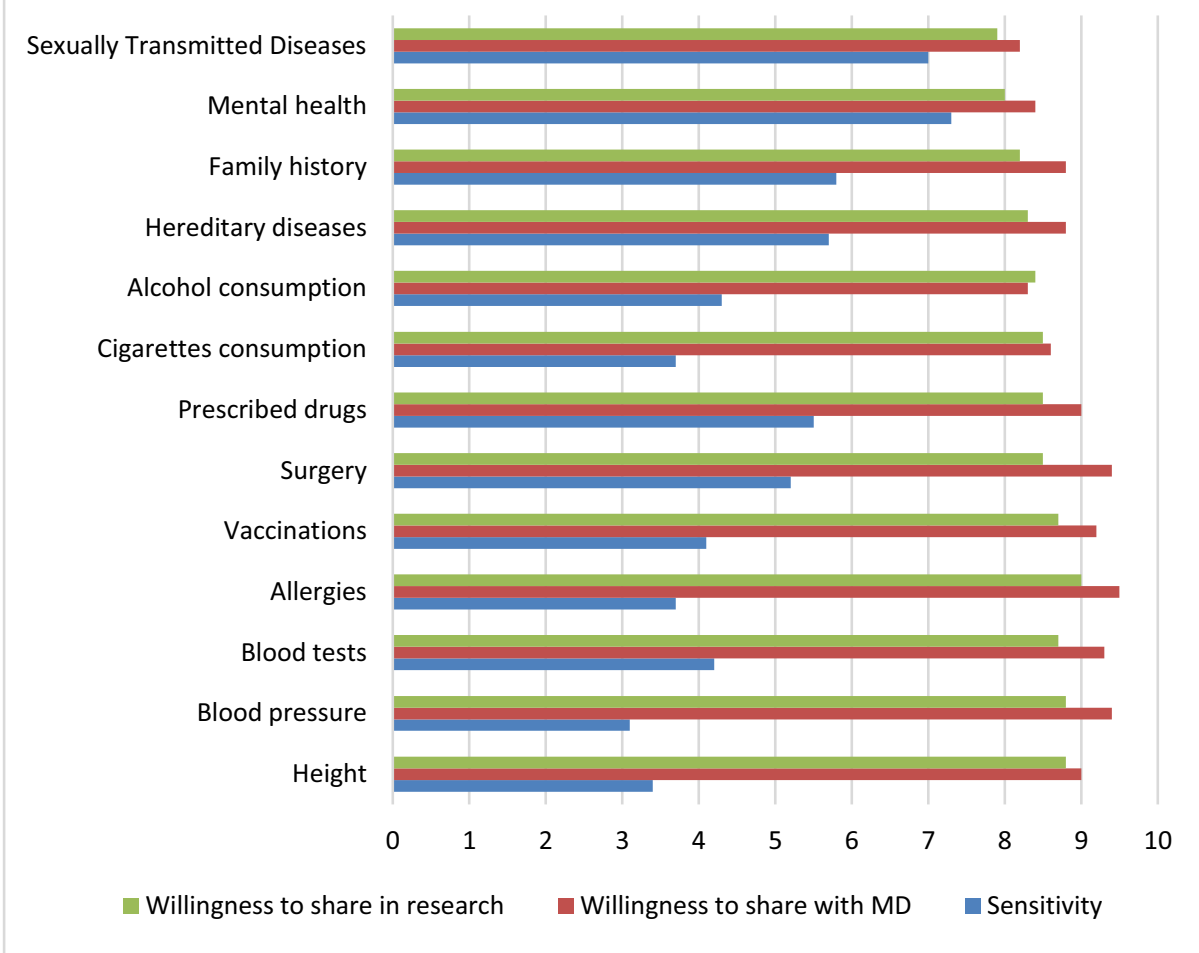

Figure 1. Sensitivity of health-related data and willingness to share

As presented in Figure 1., participants rated mental health and STD as the most sensitive health-related data followed by family history and hereditary diseases. Data with the lowest sensitivity was blood pressure, followed by height, allergies, and cigarettes consumption. Overall, participants were highly willing to share their health data with their physicians and research, with scores ranging from 7.9 for STD to 9 for allergies. They rated their trust in the anonymity of data in research on an average of 7.21, $\mathrm{SD}=2.67$.

There was a low yet significant correlation between trust in research anonymity and computer skills level $(\mathrm{r}=0.206)$ and a moderate correlation between trust in research anonymity and physician confidentiality $(\mathrm{r}=0.298)$. Health professionals $(\mathrm{M}=6.86, \mathrm{SD}=2.82)$ had significantly lower trust in doctors confidentiality compared to others $(\mathrm{M}=8.1, \mathrm{SD}=2.35)(\mathrm{t}=-2.324, \mathrm{p}=0.022)$.

\section{Discussion}

Our data showed low use of ePHR in Croatia despite awareness of its existence. In Croatia, there are several problems with the e-Health system. The first concerns the elderly population, which is not predominantly digitally literate, and the second is the lack of infrastructure due to which some households do not have Internet access. The same issues arose as the limitation of this online study. Respondents of lower 
educational status, older respondents and those from rural areas did not participate in the study. The third concern is trust in the e-Health system itself.

Our data showed that respondents believe significantly more in doctors' confidentiality than in data protection in the e-Health system. Although distrust of institutions is characteristic of post-socialist countries, it is interesting that health professionals have less trust in doctors' confidentiality than non-health workers in our sample. It raises the question of what health system employees know about the system itself that others do not. As expected, higher digital literacy was related to higher confidence in online data protection, suggesting that promoting computer skills and digital literacy could be beneficial for the broader implementation of ePHD.

As in previous study [4], the results showed that not all PHD were perceived as equally sensitive, yet the respondents were willing to share them with physicians and researchers. The interpretation of data-sharing attitudes is a complex process, related to and influenced by various factors, and involves medical ethics issues [8]. In our highly educated sample, a high willingness to share PHD might be influenced by an interest in personal health improvement or health care promotion, albeit some reserves toward trust in the e-Health system.

\section{Conclusions}

The use of the e-Health Portal in Croatia remains low, although there is a high level of trust in confidentiality, anonymity, and willingness to share data for research. Future research should involve a more diverse sample. Efforts in promoting e-Health tools could be beneficial for both the users and researchers.

\section{References}

[1] Karampela M, Ouhbi S, Isomursu M. Connected Health User Willingness to Share Personal Health Data: Questionnaire Study. JMed Internet Res. 2019 Nov; 21(11):e14537.doi:10.2196/14537

[2] Whetstone M, Goldsmith RE. Factors influencing intention to use personal health records. International Journal of Pharmaceutical and Healthcare Marketing. 2009 Apr; 3(1):8-25. doi: $10.1108 / 17506120910948485$

[3] Gille F, Brall C. Limits of data anonymity: lack of public awareness risk trust in health system activities. Life Sci Soc Policy. 2021 Jul 26;17(1):7. doi: 10.1186/s40504-021-00115-9.

[4] Helou S, Abou-Khalil V, El Helou E, Kiyono K. Factors Related to Personal Health Data Sharing: Data Usefulness, Sensitivity and Anonymity. Public Health and Informatics. 2021 May;281:1051-1055. doi:10.3233/SHTI210345.

[5] Park HS, Kim K, Soh JY, Hyun YH, Jang SK, Lee S, Hwang GY, Kim HS. Factors Influencing Acceptance of Personal Health Record Apps for Workplace Health Promotion: Cross-Sectional Questionnaire Study. JMIR Mhealth Uhealth. 2020 Jun; 8(6): e16723. doi: 10.2196/16723.

[6] Abd-Alrazaq A, Bewick BM, Farragher T, Gardner P. Factors Affecting Patients' Use of Electronic Personal Health Records' in England: Cross-Sectional Study. J Med Internet Res 2019;21(7):e12373. doi:10.2196/12373.

[7] Weng C, Friedman C, Rommel CA, Hurdle JF. A two-site survey of medical center personnel's willingness to share clinical data for research: implications for reproducible health NLP research. BMC Med Inform and Deciss Mak. 2019 Apr;19(3):70. doi:10.1186/s12911-019-0778-z.

[8] Zargaran A, Ash J, Kerry G, Rasasingam D, Gokani S, Mittal A, Zargaran D. Ethics of Smartphone Usage for Medical Image Sharing. Indian J Surg. 2018 Jun; 80(3): 300-301. doi: 10.1007/s12262-0171710-3. 